\title{
COMPORTAMIENTO DEL CONCRETO CON BAJOS PORCENTAJES DE CENIZA VOLANTE (TERMOPAIPA IV) Y AGUA CONSTANTE
}

\author{
${ }^{1}$ Luz Elena Santaella Valencia PhD \\ ${ }^{2}$ Rodrigo Salamanca Correa \\ Fecha de recepción: 2 de marzo de 2004 \\ Fecha de aprobación; 19 de abril de 2004
}

\section{RESUMEN}

Este artículo se realiza con base en el trabajo de grado titulado "Influencia de la ceniza volante procedente de Termopaipa IV adicionada en bajos porcentajes al concreto" desarrollada por los estudiantes Milena Puerta Guarín y Helbert Rivera Bernal de la Universidad Militar Nueva Granada y con patrocinio, mediante convenio con la empresa Concretos Premezclados S.A. del grupo Holcim.

Cenizas volantes provenientes de la combustión del carbón en la planta IV de Termopaipa se utilizaron para sustituir $10 \%, 20 \%$ y $30 \%$ de contenidos de cemento o de arena triturada de los diseños originales, manteniendo constante la cantidad de agua de mezclado del concreto. De los resultados obtenidos se puede deducir que el asentamiento y la densidad del concreto disminuyen cuando se sustituye arena por ceniza que cuando se reemplaza cemento por ceniza, con respecto a la muestra patrón. La sustitución del cemento por cenizas conduce a resistencias menores que al sustituir parte de la arena, manteniendo constante la cantidad de cemento y el contenido de agua. Este mismo comportamiento se observa en relación con el módulo de elasticidad del material.

Palabras clave: concreto, adiciones al concreto, cenizas, ceniza volante.

\section{SUMMARY}

This paper is based on a graduation work entitled "Influence of the addition of low percentages of fly ash, from Termopaipa IV, to concrete mix", developed by Milena

\footnotetext{
1 Ingeniera civil, coordinadora de la línea de investigación en concreto. Facultad de Ingeniería, Universidad Militar Nueva Granada, Bogotá (Colombia). Isantar@santander.umng.edu.co.

${ }^{2}$ Ingeniero civil, director de los laboratorios de la Facultad de Ingeniería, Universidad Militar Nueva Granada. Bogotá (Colombia). rsalama@santander.umng.edu.co 
Puerta Guarín and Helbert Rivera Bernal from Military Nueva Granada University (UMNG), and sponsored by Concretos Premezclados - Holcim Group company.

Fly ash, from coal combustion in IV Termopaipa Plant, was used in order to substitute 10,20 and $30 \%$ of cement or crushed sand contents of original mix designs, keeping constant the amount of water that was added. The results indicate that slump and density of concrete are strongely decreased when the fly ash substitutes sand than when it substitutes cement, respect to the reference mix. Substitution of cement by fly ash produces more decrease of strengths than substitution of crushed sand, keeping constant amount of cement and water of mix. The same performance is observed on the elasticity module of the material.

Key Words: concrete, additions to concrete, ash, fly ash

\section{INTRODUCCIÓN}

Según la definición de la norma UNE 83-415, la ceniza volante es el producto sólido y en estado de fina división, procedente de la combustión de carbón pulverizado en los hogares de centrales térmicas, que es arrastrado por los gases del proceso y recuperado de ellos, en los filtros. En esta norma, el término ceniza volante no es aplicable a los productos separados o condensados de flujos de gases procedentes de otros procesos industriales.

La ceniza volante, como subproducto, es utilizada para la fabricación de vidrio, cerámica, ladrillos, para capas de firmes de carreteras, para cama de tubos, y elaboración de hormigones. En el último caso, la ceniza volante se puede adicionar de tres formas diferentes: a) adición activa incorporada directamente en la mezcladora; b) adición inerte, es decir, como elemento sustituto o complementario de las fracciones finas de los áridos, cuando éstos son deficitarios en ellas, y c) molida conjuntamente con el clínker en la preparación de cementos tipo II, IV y V (según el Pliego RC-93) $[1,2,3]$.

\section{MÉTODO EXPERIMENTAL}

Dentro de este numeral se presentan los resultados de la caracterización de los materiales utilizados, la dosificación de las mezclas elaboradas y los resultados de su caracterización en estado fresco y endurecido.

\section{A. Materiales}

Los materiales utilizados para la elaboración de la mezcla fueron: cemento tipo III, ceniza volante, arena natural, arena triturada, gravilla de $12,5 \mathrm{~mm}$, grava de $25 \mathrm{~mm}$, 
aditivo reductor de agua y retardante Eucon R200 y agua.

Cemento: se utilizó cemento tipo III, ARI suministrado por Cementos Boyacá. Su caracterización se realizó con los siguientes ensayos: peso específico, finura, consistencia normal, tiempo de fraguado y resistencia a la compresión, cuyos resultados promedios de ocho repeticiones aparecen en la tabla $\mathrm{N}^{0} 1$.

Tabla № 1. Caracterización del cemento tipo III.

\begin{tabular}{|l|r|}
\hline \multicolumn{1}{|c|}{ PARÁMETRO } & \multicolumn{1}{|c|}{ VALOR } \\
\hline Peso especifico, $\mathrm{gr} / \mathrm{cm}^{3}$ & 3,16 \\
\hline Finura Blaine, $\mathrm{m}^{2} / \mathrm{kg}$ & 3893 \\
\hline Consistencia normal (\%) & 27,8 \\
\hline T. de fraguado inicial (minutos) & 79 \\
\hline T. de fraguado final (minutos) & 110 \\
\hline \multirow{3}{*}{ Resistencia a la compresión $\left(\mathrm{kg} / \mathrm{cm}^{2}\right)$} & 3 días $=317$ \\
\cline { 2 - 2 } & 7 días $=357$ \\
\cline { 2 - 2 } & 28 días $=511$ \\
\hline
\end{tabular}

Ceniza volante: en el proceso de generación de energía de la planta IV de Termopaipa se producen, como residuo de la combustión del carbón, dos tipos de cenizas: las cenizas de fondo, que caen en el cenicero de la cámara de combustión, donde se mezclan con agua y se retiran del sistema y las cenizas volantes, que salen con los gases de la cámara por los ductos de conducción, captadas a la salida de la chimenea mediante filtros electrostáticos; de allí pasan al tanque de cenizas y son las utilizadas para adicionarlas al concreto en este trabajo. La composición química de la ceniza volante se presenta en la Tabla 2.

Tabla $\mathbf{N}^{\circ}$ 2. Características de la ceniza volante.

\begin{tabular}{|c|r|r|r|r|r|r|r|r|r|r|r|r|}
\hline \multicolumn{10}{|c|}{ PORCENTAJE DE ÓXIDOS } \\
\hline $\mathrm{SiO}_{2}$, & $\mathrm{Al}_{2} \mathrm{O}_{3}$ & $\mathrm{Fe}_{2} \mathrm{O}_{3}$ & $\mathrm{TiO}_{2}$ & $\mathrm{CaO}$ & $\mathrm{MgO}$ & $\mathrm{Na}_{2} \mathrm{O}$ & $\mathrm{K}_{2} \mathrm{O}$ & $\mathrm{SO}_{3}$ & $\mathrm{P}_{2} \mathrm{O}_{5}$ & $\mathrm{BaO}$ & $\mathrm{SrO}$ & $\mathrm{MnO}$ \\
\hline 61,3 & 23,5 & 4,2 & 1,2 & 1,0 & 0,73 & 0,53 & 1,27 & 0,31 & 0,68 & 0,15 & 0,2 & 0,02 \\
\hline Inquemados $=7,6 \%$ & \multicolumn{10}{|c|}{ Peso específico $=2,16 \mathrm{gr} / \mathrm{cm}^{3}$} \\
\hline
\end{tabular}

Agregados: los agregados empleados en el diseño y elaboración de la mezcla corresponden a una grava con tamaño máximo nominal de $25 \mathrm{~mm}$, una gravilla con tamaño máximo nominal de $12.5 \mathrm{~mm}$, arena natural y arena triturada, de la línea 2 de la Planta de INGENIESA, y que se extraen de la mina de agregados de Manas emplazada en el sector de Tunjuelito al sur de la ciudad de Bogotá. Los ensayos realizados para la caracterización de los agregados fueron: granulometría, densidad y absorción, masas unitarias y humedad. El valor promedio de las ocho repeticiones de cada ensayo se presenta en la Tabla $N^{\circ} 3$ y los resultados de las granulometrías de los agregados se presentan en la Tabla $\mathrm{N}^{\circ} 4$.

Tabla 3. Resumen de resultados de ensayos del agregado.

\begin{tabular}{|l|c|c|c|c|}
\hline Parámetros & Grava & Grava & Arena & Arena \\
\hline
\end{tabular}




\begin{tabular}{|l|c|c|c|c|}
\hline & $\mathbf{2 5 m m}$ & $\mathbf{1 2 . 5} \mathbf{m m}$ & Natural & Triturada \\
\hline Densidad aparente, $\mathrm{gr} / \mathrm{cm}^{3}$ & 2,45 & 2,45 & 2,53 & 2,44 \\
\hline Densidad (sss), gr/cm & 3 & 2,51 & 2,57 & 2,49 \\
\hline Densidad nominal, $\mathrm{gr} / \mathrm{cm}^{3}$ & 2,50 & 2,61 & 2,65 & 2,57 \\
\hline Absorción, \% & 1,93 & 2,45 & 1,85 & 2,10 \\
\hline Masa unitaria suelta, $\mathrm{kg} / \mathrm{m}^{3}$ & 1387 & 1345 & 1489 & 1403 \\
$\%$ Vacíos & 43 & 45 & 41 & 42 \\
\hline Masa unitaria compacta, $\mathrm{kg} / \mathrm{m}^{3}$ & 1451 & 1460 & 1584 & 1571 \\
$\%$ Vacíos & 41 & 40 & 37 & 36 \\
\hline Módulo de finura & 7,55 & 5,78 & 2,71 & 2,89 \\
\hline
\end{tabular}

Tabla 4. Análisis granulométrico promedio de los agregados.

\begin{tabular}{|c|c|c|c|c|c|}
\hline \multicolumn{6}{|c|}{ PORCENTAJE QUE PASA } \\
\hline \multicolumn{2}{|c|}{ TAMICES } & \multicolumn{2}{|c|}{ GRAVA } & \multicolumn{2}{|c|}{ ARENA } \\
\hline \# & $\mathbf{m m}$ & $25 \mathrm{~mm}$ & $12.5 \mathrm{~mm}$ & Natural & Triturada \\
\hline 3" & 76.2 & 100.0 & 100.00 & 100.0 & 100.0 \\
\hline $1^{1 / 2^{2}}$ & 38.10 & 99.57 & 100.00 & 100.0 & 100.0 \\
\hline $3 / 4 "$ & 19.00 & 75.26 & 100.00 & 100.0 & 100.0 \\
\hline $1 / 2 "$ & 12.00 & 37.90 & 99.06 & 100.0 & 100.0 \\
\hline $3 / 8 "$ & 9.51 & 18.65 & 81.49 & 100.0 & 100.0 \\
\hline \#4 & 4.76 & 5.05 & 23.76 & 98.99 & 99.50 \\
\hline \# 8 & 2.38 & 2.58 & 6.70 & 77.73 & 76.23 \\
\hline \# 16 & 1.19 & 2.05 & 3.76 & 60.17 & 57.47 \\
\hline \# 30 & 0.590 & 1.86 & 3.04 & 49.16 & 44.17 \\
\hline \# 50 & 0.297 & 1.47 & 2.34 & 32.51 & 23.23 \\
\hline$\# 100$ & 0.149 & 0.76 & 1.42 & 10.64 & 9.80 \\
\hline \# 200 & 0.074 & 0.34 & 0.90 & 4.76 & 5.53 \\
\hline Fondo & & 0.0 & 0.00 & 0.0 & 0.0 \\
\hline
\end{tabular}

Aditivo: se usó el aditivo EUCON R200 o Concreplast, que es un reductor de agua y retardador de fraguado, fabricado por Toxement, el cual cumple con las normas ASTM C494 tipo D y NTC1299.

\section{B. Proporción de la mezcla.}

Se procedió al diseño de las mezclas de concreto, fijando de antemano el contenido de agua en 179 litros $/ \mathrm{m}^{3}$ para conseguir un asentamiento entre 7 y $9 \mathrm{~cm}$, y el contenido de cemento en $250 \mathrm{~kg} / \mathrm{m}^{3}$ para obtener una resistencia especificada de $210 \mathrm{~kg} / \mathrm{cm}^{2}$, datos sugeridos por la empresa Concretos Premezclados. Por lo tanto, la cantidad total de agregados se determinó con el método del volumen absoluto al restar del volumen total de la mezcla $\left(1.01 \mathrm{~m}^{3}=1010\right.$ litros) los volúmenes de cemento y agua. En la tabla $\mathrm{N}^{\circ} 5$ se observan las proporciones definitivas de las mezclas del diseño patrón (DP), los diseños que sustituyen el cemento por ceniza volante (D1=10\%, D2=20\% y D3=30\%) y por último están los diseños que sustituyen la arena triturada por ceniza volante (D4=10\%, D5=20\% y D6=30\%) manteniendo constante la cantidad de cemento.

Tabla 5. Dosificaciones utilizadas para los diseños de mezclas.

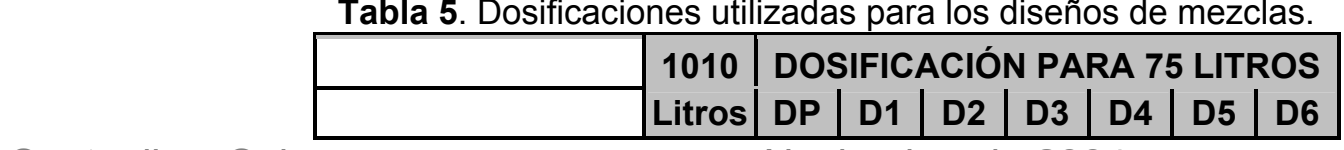




\begin{tabular}{|l|c|r|r|r|r|r|r|r|} 
Grava 25mm $(\mathrm{kg})$ & 780 & 57.9 & 57.9 & 57.9 & 57.9 & 57.9 & 57.9 & 57.9 \\
\hline Gravilla 12.5mm (kg) & 334 & 24.8 & 24.8 & 24.8 & 24.8 & 24.8 & 24.8 & 24.8 \\
\hline Arena natural $(\mathrm{kg})$ & 483 & 35.9 & 35.9 & 35.9 & 35.9 & 35.9 & 35.9 & 35.9 \\
\hline Arena triturada $(\mathrm{kg})$ & 260 & 19.3 & 19.3 & 19.3 & 19.3 & 17.4 & 15.6 & 13.7 \\
\hline Cemento tipo III (kg) & 250 & 18.6 & 16.7 & 14.9 & 13.0 & 18.6 & 18.6 & 18.6 \\
\hline Ceniza volante (kg) & 0 & 0.0 & 1.9 & 3.7 & 5.6 & 1.9 & 3.7 & 5.6 \\
\hline Agua (kg) & 179 & 13.3 & 13.3 & 13.3 & 13.3 & 13.3 & 13.3 & 13.3 \\
\hline Eucon R200 (gr) & 65.0 & 65.0 & 65.0 & 65.0 & 65.0 & 65.0 & 65.0 & 65.0 \\
\hline
\end{tabular}

El contenido del aditivo reductor de agua y retardante EUCON R200 fue de 0,35\% del peso de cemento, el cual se mantuvo constante (65gr. para 75 litros de concreto) en todas las mezclas. Los 75 litros de cada mezcla de concreto fueron elaborados en un trompo con capacidad para 170 litros, en un tiempo de mezclado de 4 minutos.

\section{Ensayos}

Para determinar el comportamiento en estado fresco de cada mezcla de concreto se realizaron los siguientes ensayos: control de temperatura, asentamiento y masa unitaria del concreto. Mientras que los ensayos realizados para determinar las características del concreto en estado endurecido fueron: resistencia a la compresión para lo cual se elaboraron seis cilindros de $15 \mathrm{~cm} \times 30 \mathrm{~cm}$ para ensayar por pares a 14, 28 y 56 días de edad; el módulo de elasticidad se determinó en cada caso sobre dos cilindros de $15 \mathrm{~cm}$ x $30 \mathrm{~cm}$ a las edades de 28 y 56 días. Todos los cilindros fueron desmoldados a las 24 horas de elaborados y almacenados en un cuarto de curado con humedad y temperatura controladas hasta la respectiva edad de ensayo.

\section{DISCUSIÓN DE RESULTADOS}

Los resultados de los ensayos mostrados en la tabla $N^{\circ} 6$, corresponden a los promedios de ocho repeticiones realizadas a las mezclas de concreto en estado fresco y endurecido.

Tabla № 6. Resultados promedios de ensayos realizados al concreto sin adición, con sustitución de cemento por ceniza volante, y con sustitución de arena por ceniza volante.

\begin{tabular}{|c|c|c|c|c|c|c|c|}
\hline \multirow{3}{*}{ MEZCLAS } & \multirow{2}{*}{\multicolumn{2}{|c|}{ CONCRETO FRESCO }} & \multicolumn{5}{|c|}{ CONCRETO ENDURECIDO } \\
\hline & & & \multicolumn{3}{|c|}{$\begin{array}{c}\text { Resistencia a la compresión } \\
\left(\mathrm{kg} / \mathrm{cm}^{2}\right)\end{array}$} & \multicolumn{2}{|c|}{$\begin{array}{c}\text { Módulo elástico } \\
\left(\mathrm{kg} / \mathrm{cm}^{2}\right)\end{array}$} \\
\hline & $\begin{array}{l}\text { Fluidez, } \\
\text { cm }\end{array}$ & $\begin{array}{c}\text { Densidad } \\
\mathrm{Kg} / \mathrm{m}^{3}\end{array}$ & 14 días & 28 días & 56 días & 28 días & 56 días \\
\hline DP: $0 \% C V+100 \% C$ & 8.7 & 2340 & 316 & 321 & 345 & 169827 & 193613 \\
\hline D1: $10 \% C V+90 \% C$ & 5.4 & 2334 & 303 & 344 & 356 & 177637 & 201165 \\
\hline D2: $20 \% C V+80 \% C$ & 5.3 & 2321 & 267 & 300 & 320 & 174204 & 195697 \\
\hline
\end{tabular}




\begin{tabular}{|l|l|l|l|l|l|l|l|}
\hline D3: $30 \% C V+70 \% C$ & 4.7 & 2313 & 195 & 247 & 279 & 160171 & 179170 \\
\hline D4: $10 \% C V+90 \% A T$ & 4.9 & 2337 & 332 & 367 & 405 & 183033 & 195280 \\
\hline D5: $20 \% C V+80 \% A T$ & 3.8 & 2321 & 357 & 401 & 452 & 184413 & 201967 \\
\hline D6: $30 \% C V+70 \% A T$ & 3.5 & 2310 & 353 & 395 & 448 & 183536 & 203425 \\
\hline
\end{tabular}

El diseño patrón (DP) sin ningún porcentaje de adición, presenta mayor fluidez, respecto a las mezclas con agua constante y sustituciones de 10, 20 o $30 \%$ de cemento y arena por ceniza volante; en consecuencia, a mayor cantidad de ceniza menor es la fluidez.

El diseño patrón (DP) presenta la mayor densidad, la cual va disminuyendo con el aumento del porcentaje adicionado de ceniza volante, tal como se observa en la densidad de las mezclas con sustitución de $30 \%$ de ceniza volante por cemento (D3) y arena (D6), en las cuales la disminución es de $27 \mathrm{~kg} / \mathrm{m}^{3}$, cuando se sustituye cemento y de $30 \mathrm{~kg} / \mathrm{m}^{3}$ para la sustitución de arena, respecto al diseño patrón (DP).

A la edad de 28 y 56 días, la resistencia a la compresión es superior al diseño patrón (DP) cuando se reemplaza $10 \%$ de cemento por ceniza volante; esto también sucede cuando se reemplaza la arena triturada por $10 \%, 20 \%$ y $30 \%$ de ceniza volante a 14 , 28 y 56 días. La resistencia a la comprensión es mayor en este último caso, con incrementos de $37 \mathrm{~kg} / \mathrm{cm}^{2}$ a 14 días, $74 \mathrm{~kg} / \mathrm{cm}^{2}$ a 28 días y $103 \mathrm{~kg} / \mathrm{cm}^{2}$ a 56 días, para la sustitución de $30 \%$ de ceniza volante con respecto al diseño patrón (DP).

Los diseños (D1 y D2) con $10 \%$ y $20 \%$ de ceniza volante en reemplazo de cemento incrementan su módulo de elasticidad con respecto al diseño patrón (DP) excepto el diseño con $30 \%$ de ceniza volante (D3). Mientras que todas las mezclas (D4, D5 y D6) con adiciones de cenizas (CV) en reemplazo de arena triturada (AT) presentan un módulo de elasticidad mayor al diseño patrón.

\section{CONCLUSIONES}

La ceniza volante disminuye el asentamiento sin afectar la "trabajabilidad" de ella. Es menos fluida la mezcla con sustituciones de ceniza por arena triturada que la mezcla con sustituciones de cenizas por cemento.

La sustitución de ceniza por cemento disminuye la masa unitaria de las mezclas en menor proporción que la adición de ceniza por arena triturada. Por lo tanto, las cenizas volantes contribuyen a la reducción de la masa por metro cúbico de concreto fresco, haciéndolas más livianas en comparación con el concreto patrón.

La mejor dosificación para la sustitución de cemento por ceniza volante es la de $10 \%$, debido a que los resultados de los ensayos de caracterización de la mezcla fueron los 
mejores respecto de las demás variaciones de este tipo. La mejor dosificación para la sustitución de arena triturada por ceniza volante es la de $20 \%$, debido a que los resultados de la caracterización de la mezcla mantuvieron un crecimiento progresivo en la mezcla con respecto a las otras variaciones de este tipo.

El concreto adquiere resistencias altas con pequeñas adiciones de ceniza en remplazo de cemento a partir de 28 días. Las sustituciones de arena triturada por ceniza adquieren mayores resistencias a medida que se incrementa la cantidad de ceniza adicionada y a medida que aumenta la edad, debido a que la cantidad de cemento permanece constante.

Por otra parte, el concreto adquiere mayor módulo de elasticidad cuando disminuyen las sustituciones de ceniza volante $(10 \%$ y $20 \%)$ por cemento en las mezclas. Pero en las mezclas que mantienen la misma cantidad de cemento $(18,56 \mathrm{~kg})$ y se remplaza arena triturada por ceniza volante se aumentan los valores del módulo de elasticidad, tal como sucede con la resistencia a la compresión.

\section{BIBLIOGRAFÍA}

[1] Ramírez j. l., Alonso (1990). Orígenes, tipos y caracterización de las cenizas volantes. Madrid. Centro de Estudio y Experimentación de Obras Públicas, Cuaderno de Investigación, $67 \mathrm{p}$.

[2] Asociación de Investigaciones Industrial Eléctrica (ASINEL), (1982). Las cenizas volantes y sus aplicaciones. Madrid.

[3] García Rosselló, J. (1987). Las cenizas volantes en los hormigones para presas. Madrid. 